\title{
Genomic Loci Evaluation with Albuminuria in New-Onset Insulin Dependent Diabetic Patients
}

\author{
Anna Sleder ${ }^{1}$, Jia Li ${ }^{2}$, Andrea Cassidy-Bushrow ${ }^{3}$, Sharon Cresci ${ }^{4}$, Keoki Williams ${ }^{5}$, Hani N Sabbah ${ }^{6}$ and David Lanfear ${ }^{7 *}$ \\ ${ }^{1}$ Department of Internal Medicine, Henry Ford Hospital, Detroit, Michigan, USA \\ ${ }^{2}$ Electrical and Computer Engineering Department, Oakland University, Rochester, Michigan, USA \\ ${ }^{3}$ Department of Public Health Sciences, Henry Ford Hospital Detroit, Michigan, USA \\ ${ }^{4}$ Cardiovascular Division, Washington University School of Medicine, St. Louis, Missouri, USA \\ ${ }^{5}$ Center for Health Services Research, Henry Ford Hospital, Detroit, Michigan, USA \\ ${ }^{6}$ Cardiovascular Research Laboratories Henry Ford Hospital, Detroit, Michigan, USA \\ ${ }^{7}$ Heart and Vascular Institute, Henry Ford Hospital, Detroit, Michigan, USA
}

\begin{abstract}
Background: Extensive data supports the genetic underpinnings of diabetes and recent studies implicate several genetic loci associated with renal insufficiency and albuminuria. Moreover, albuminuria in diabetic patients is an important risk marker for atherosclerotic disease as well as cardiomyopathy. The purpose of this study was to identify genetic determinants of albuminuria in a diabetic patient population at the time of insulin initiation.

Methods: Study population included type 2 diabetic subjects at the time of initiation of insulin in an observational cohort who donated saliva samples for DNA extraction. Urine albumin to creatinine ratio (UACR) and HbA1c levels at baseline were collected and analyzed for association with genotype $(\mathrm{N}=128)$.

Results: Although none of the SNPs met statistical significance after Bonferroni adjustment, two regions showed near-significant associations with UACR; one on chromosome $1(p=8.66 \mathrm{E}-07)$ and one on chromosome $12(p=9.82$ E-07).

Conclusions: In this small study of newly insulin-dependent diabetics we identified two genomic regions possibly associated with albuminuria. These loci are good candidates for further investigation into the moderators of cardiovascular disease in diabetics. Larger confirmatory studies are needed.
\end{abstract}

Keywords: Genetics; Loci; Albuminuria; Diabetes

\section{Introduction}

Cardiovascular disease (CVD) accounts for the vast majority of morbidity and mortality in the diabetic patient population [1]. In 1999 diabetes was recognized as a coronary heart disease equivalent [2] and more recently it has been well recognized that the presence of diabetes increases the risk of heart failure by two to four fold [3]. Moreover, among diabetic and cardiovascular patients, renal dysfunction is common, and associated with a worse prognosis [4,5]. In this setting, albuminuria is not only a marker of renal disease but is also an important risk factor for CVD progression [6] and more recently it has been recognized as a risk factor for the development of heart failure [7]. While tight glycemic control reduces micro vascular complications, similar benefits in terms of CVD are not clear making early genetic susceptibility identification potentially clinically beneficial [8]. Thus, identifying the contributing factors which underlie CVD progression in patients with diabetes remains an important question in order to better anticipate risk and develop effective interventions.

Genetic factors may help explain some of the variability in diabetic CVD development, and genomic studies in this setting could help identify potential pathways to intervene upon. Genome wide association studies (GWAS) have already identified genetic loci related to reduce glomerular filtration rates (GFR) among patients with diabetes [9] but parallel efforts to examine diabetic CVD development or its risk factors are less common. For example GWAS have identified 16 loci for the estimated glomerular filtration rate (eGFR) in European populations and seven eGFR-associated single nucleotide polymorphisms (SNPs) from persons of African ancestry from the CKDGen Consortium and the CARe Renal Consortium, respectively $[10,11]$.

Significant genetic loci of UMOD, GCKR, and SHROOM3 in renal complications among diabetic patients have been previously described in various population groups $[12,13]$. Since diabetes is an important risk factor for atherosclerosis and CVD, there is great importance of early identification and control. Microalbuminuria, a marker of renal disease, in the diabetic patient population is a poor prognostic indicator for CVD [14]. Therefore, the use and association of albuminuria as a target for further genetic investigations remains. Further, in the diabetic population, the use of insulin marks a severity disease where insulin resistance is high and disease is generally poorly controlled [15].

Identifying diabetics with a genetic predisposition to reno-vascular disease prior to the onset of chronic kidney disease (CKD) and CVD could lead to prevention of morbidity and reduce mortality among this population. This demonstrates the value of this strategy but additional data specific to albuminuria is needed. Common genetic loci have been studied in terms of association to albuminuria but no association has been found [16]. Despite this accumulating knowledge, the genetic and pathologic mechanisms that determine development and progression of CVD remain largely unknown [17].

*Corresponding author: David Lanfear, Heart and Vascular Institute, Henry Ford Hospital, 2799 W. Grand Blvd., K14, Detroit MI, 48202, USA, Tel: (313) 916-6375; Fax: (313) 916-8799; E-mail: dlanfea1@hfhs.org

Received October 25, 2013; Accepted January 22, 2014; Published January 29 2014

Citation: Sleder A, Cassidy-Bushrow A, Cresci S, Williams K, Sabbah HN et al. (2014) Genomic Loci Evaluation with Albuminuria in New-Onset Insulin Dependent Diabetic Patients. J Pharmacogenomics Pharmacoproteomics 5: 122. doi:10.4172/2153-0645.1000122

Copyright: $(2014$ Sleder A, et al. This is an open-access article distributed under the terms of the Creative Commons Attribution License, which permits unrestricted use, distribution, and reproduction in any medium, provided the original author and source are credited. 
Citation: Sleder A, Cassidy-Bushrow A, Cresci S, Williams K, Sabbah HN, et al. (2014) Genomic Loci Evaluation with Albuminuria in New-Onset Insulin Dependent Diabetic Patients. J Pharmacogenomics Pharmacoproteomics 5: 122. doi:10.4172/2153-0645.1000122

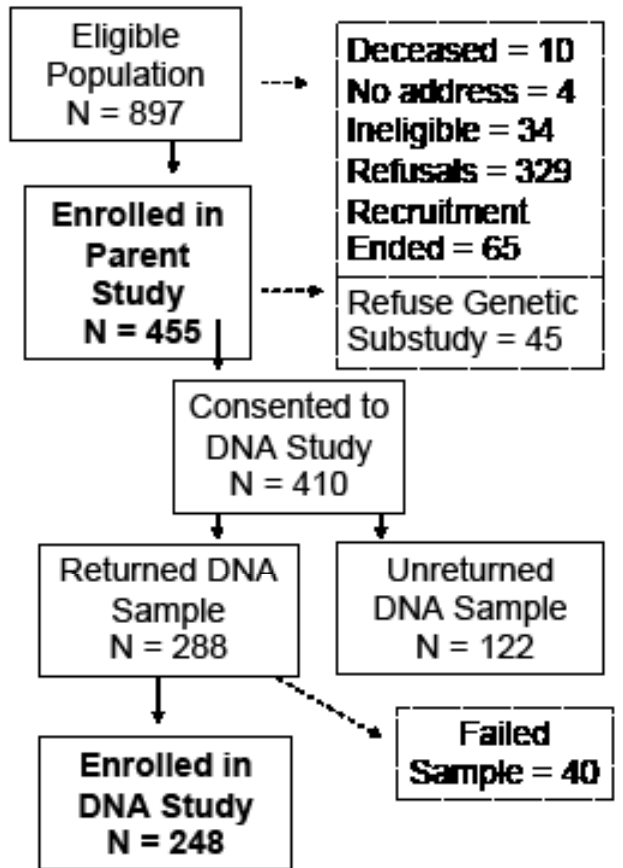

Figure 1: Study Population derived from Parent Study: CREDIT.

We sought to identify genetic determinants of albuminuria in diabetic patients recently initiated on insulin therapy. Acknowledging the great complexity of diabetes as a disease state, the use of insulin as a pre-requisite for inclusion criteria was aimed at evaluating a population with a similar disease process and severity. Our study team leveraged data collected as part of an ongoing observational study of diabetic patients who initiated insulin therapy (Long-Term International Non-Interventional Study in People with Type 2 Diabetes Treated with Insulin: CREDIT) (Figure 1), collecting blood samples for DNA isolation, obtaining hemoglobin A1c (HbAlc), and urine collection for albumin and creatinine measures from the electronic medical record (EMR).

\section{Methods}

The study was approved by the institutional review board of Henry Ford Hospital and all subjects provided written informed consent. The study was a single center retrospective cohort study of patients with diabetes who had first time initiation of insulin. Subjects were initially identified using EMR including claims-data indicating the initiation of insulin therapy within the previous 5 years and were recruited by telephone, then followed prospectively for up to two years as part of parent study involving recruitment of a cohort of Type 2 diabetic patients.

Patients who were identified as having type 2 DM (ICD 9 codes: 250.xx, 357.2x, 362.0x, 366.41), and who were treated with insulin for at least one month, were $>40$ years of age, and had a HbAlc measure within 3 months prior to starting insulin treatment were identified. Patients who initiated insulin between January 1, 2005 and October 31, 2009 were eligible. Those eligible were contacted via phone and once entered into the parent study were asked if they would also like to participate in the genetic sub-study. Among a total of 450 subjects recruited into the parent study, 248 consented to genetic analysis and provided a suitable sample for DNA extraction [18]. UACR and HbAlc levels within 90 days of insulin initiation date were collected from the EMR and considered baseline.

Genetic material was collected using saliva DNA isolation kits (DNA Genotek). After verbal consent was obtained, saliva kit and written consent form was mailed to the potential subject. Only samples that were accompanied by signed consent form were used in the study. Genotyping was accomplished using the CardioMetaboChip from Allumina ${ }^{\infty}$ which types approximately 250,000 loci, many of which have previous associations with cardiac, metabolic, or anthropometric phenotypes. As quality control the SNPs with a call rate less than $90 \%$, those with minor allele frequency of less than $1 \%$, and samples with call rate less than $90 \%$ were excluded. This resulted in 248 evaluable samples.

To test for the association between UACR and genotype, $\log 10$ transformation was first applied due to non-normal distribution. Following log transformation, linear mixed models that account for pairwise genotypic similarity of individuals, using efficient mixedmodel association eXpedited (EMMAX), were used to test for association and population structure [19]. Models were adjusted for the first principal component to infer population structure and avoid spurious findings due to population stratification. Baseline $\mathrm{HbAlc}$ was also adjusted for utilizing first principle component in order to account for disease severity. The Q-Q plot (Figure 2) demonstrates the effectiveness of the adjustment for UACR with first principle component. Multiple comparisons were accounted for by Bonferroni correction with a critical P-value of $4.5 \times 10^{-7}$.

\section{Results}

After the DNA extraction, genotyping, quality control, and database merging were completed there were roughly 60 million individual genotypes, and 248 met all quality standards. Among those samples, 15 subjects had missing data on HbAlc and 109 subjects had missing data on UACR. All data including baseline (within 3 months of insulin initiation) UACR was available for 124 subjects. Demographic and baseline characteristics of the study population with complete data are presented in Table 1.

The primary results are depicted in Figure 3, a Manhattan plot

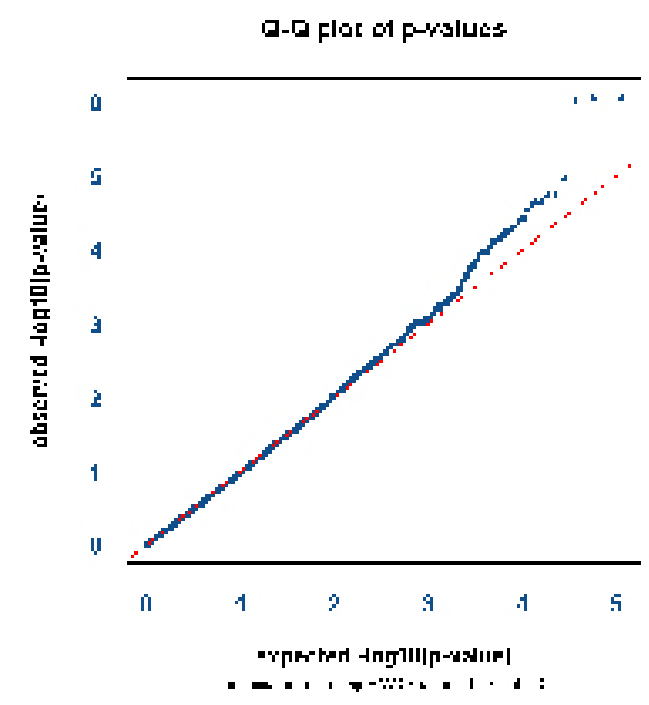

Figure 2: Q-Q Plot of Observed vs. Expected P-values of UACR with principle component adjustment $(\mathrm{N}=124)$. 
Citation: Sleder A, Cassidy-Bushrow A, Cresci S, Williams K, Sabbah HN, et al. (2014) Genomic Loci Evaluation with Albuminuria in New-Onset Insulin Dependent Diabetic Patients. J Pharmacogenomics Pharmacoproteomics 5: 122. doi:10.4172/2153-0645.1000122

\begin{tabular}{|c|c|}
\hline Characteristic & Value \\
\hline Age at initial enrollment & $64.9 \pm 10.3$ \\
\hline Gender & Male $51 \%$ \\
\hline Race & Female 49\% \\
\hline African-American Caucasian Other & $42.9 \%$ \\
\hline BMI $\geq 30 \mathrm{~kg} / \mathrm{m}^{2}$ & $54.5 \%$ \\
\hline HbA1c (\%) & $2.6 \%$ \\
\hline Hypertension & $68.9 \%$ \\
\hline Duration of DM (years) & $8.0 \pm 1.6$ \\
\hline Hyperlipidemia & $96.9 \%$ \\
\hline
\end{tabular}

Table 1: Study population characteristics $(\mathrm{N}=124)$.

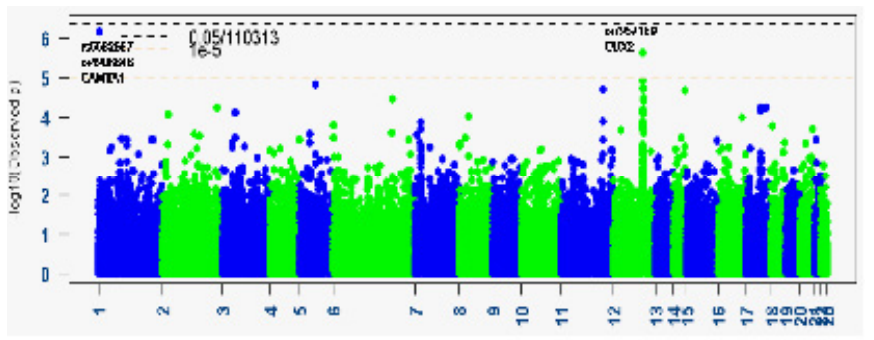

Figure 3: Manhattan Plot of Urine Albumin to Creatinine ratio (adjusted for $\mathrm{PC} 1$ and $\mathrm{HbA} 1 \mathrm{c})$

\begin{tabular}{|c|c|c|c|c|c|}
\hline CHR & SNP 10 & $\begin{array}{c}\text { parameter } \\
\text { estimate }\end{array}$ & P value & Gene & Type \\
\hline 1 & rs6682687 & -0.4362 & $8.66 \mathrm{E}-07$ & CAMTA1 & Intron \\
\hline 1 & & -0.4362 & $8.66 \mathrm{E}-07$ & CAMTA1 & intron \\
\hline 12 & rs7957169 & -0.3459 & $9.82 \mathrm{E}-07$ & CUX2 & Intron \\
\hline 12 & rs10219736 & -0.3313 & $2.36 \mathrm{E}-05$ & CUX2 & near-gene \\
\hline 12 & rs3803167 & -0.3179 & $4.94 \mathrm{E}-05$ & CUX2 & coding- synonymous \\
\hline 12 & rs7398343 & -0.2953 & $5.92 \mathrm{E}-05$ & CUX2 & intron \\
\hline 12 & rs2339718 & -0.3118 & $7.22 \mathrm{E}-05$ & CUX2 & intron \\
\hline 12 & rs12423926 & -0.3117 & $7.64 \mathrm{E}-05$ & CUX2 & Intron \\
\hline
\end{tabular}

Table 2: SNPs with near-significant $P$-values associated with albuminuria.

of the genomic coordinate along the $\mathrm{X}$-axis with associated $P$-value of UACR for each SNP displayed on the Y axis. Although none of the SNPs passed genome-wide significance after adjusting for multiple testing using the Bonferroni adjustment (Table 2), there were two regions with near-significant associations. The first, on chromosome 1, included two SNPs, rs6682687 and rs4908646 in the gene calmodulinbinding transcription activator 1 (CAMTA1) and showed a $\mathrm{p}=8.6 \times 10^{-7}$. The other region was on chromosome 12 , with the lowest $\mathrm{p}=9.8 \times 10^{-7}$ for rs7957169. Interestingly, this site included a large number of nearby sequence variants with a range of $\mathrm{p}$ values in an association spike. This peak is quite broad, with its base being roughly $300 \mathrm{~kb}$ (Figure 4).

\section{Discussion}

In this small study we have identified candidate genes for UACR/ albuminuria in diabetics. Since albuminuria is an important marker of cardiovascular disease, specifically of atherosclerosis and heart failure, these are thus candidate genes for the progression of CVD in diabetics. There remains a critical public health need to better understand the pathophysiology of diabetes in terms of cardiovascular complications and knowledge of the genetic underpinnings should further this goal. Acknowledging the complexity of disease states of DM, CVD and CKD confounded by the interplay of comorbid conditions compounds the difficulty in analysis. Our data is the first that we know of to identify genetic factors contributing to albuminuria timed to the onset of insulin use in diabetics. The use of insulin initiation as a marker of disease severity, with presumed insulin resistance prompting initiation, allows for a more focused population of study. These findings are consistent with and add to the existing data. Work by Ellis et al. [13] tested genetic loci previously associated with eGFR for association with albuminuria but did not find significant loci. Findings in Deshmukh et al. [9], with confirmation of the existence of eGFR-associated SNPs (UMOD, GCKR, and SHROOM3) elucidated the importance of correcting for albuminuria presence as it changed gene effect within the study; this suggests that further elucidation of a gene for albuminuria is warranted with consideration of eGFR. Larger studies aimed at a disease-focused population, such as diabetic patients with recent insulin initiation, is an avenue for further evaluation.

The two genomic loci that we found to be associated with albuminuria on chromosomes 1 and 12 did localize within specific gene regions. On chromosome 1 , two SNPs of interest $\left(\mathrm{p}=8.6 \times 10^{-7}\right)$ were within introns of calmodulin-binding transcription activator 1 (CAMTA1, rs6682687 and rs4908646). These particular variants did not have any prior reported clinical associations that we could identify despite other SNPs within the CAMTA1 gene with GWAS associations to heart failure and coronary artery disease (rs12740374 and rs4908443, respectively) $[20,21]$. The chromosome $12 \mathrm{SNP}$ of interest (rs7957169, $\mathrm{p}=9.8 \times 10^{-7}$ ) was at the tip of broad peak of association, spanning roughly $300 \mathrm{~kb}$. The most prominent gene in the peak is the cut-like homeobox 2 (CUX2) gene. The CUX2 gene region has recently been associated with Type 1 diabetes in the 1000 Genomes Project using data from the WellcomeTrust Case Control Consortium [22]. To our knowledge this is its first association with albuminuria. In addition there are other nearby genes (Figure 4) that should also be considered. Additional studies are needed to confirm and extend these preliminary findings. Fortunately, there are ongoing, large meta-analyses of type 2 diabetes studies (using genotype imputation via the 1000 Genomes reference panel) underway which hopefully will shed further light on this important phenotype [23].

Our data should be interpreted in view of some limitations. This was a small study without a validation set, so these findings should be regarded as preliminary and require further confirmation. This was an observational cohort thus selection bias is always a concern; however

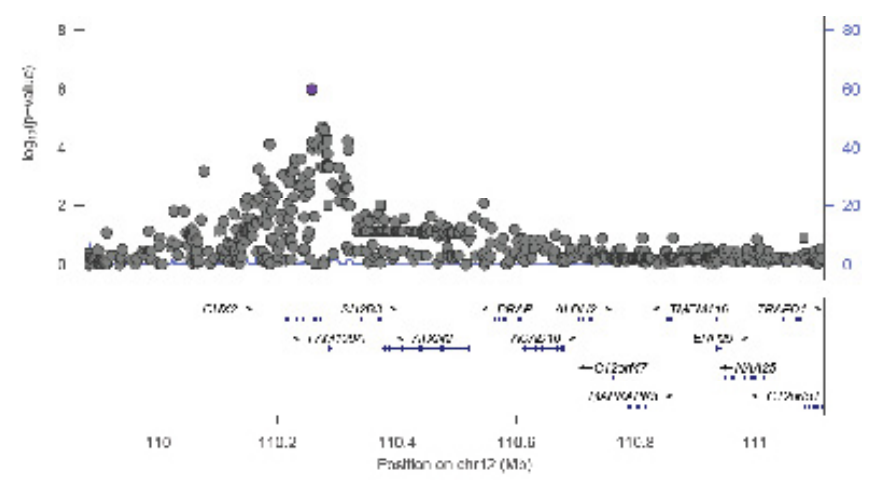

Figure 4: Urine albumin to creatinine ratio with position on chromosome 12. 
Citation: Sleder A, Cassidy-Bushrow A, Cresci S, Williams K, Sabbah HN, et al. (2014) Genomic Loci Evaluation with Albuminuria in New-Onset Insulin Dependent Diabetic Patients. J Pharmacogenomics Pharmacoproteomics 5: 122. doi:10.4172/2153-0645.1000122

the investigators put great effort into recruiting all potential subjects who met enrollment criteria. Moreover there could be selection bias on the part of the subjects since they could volunteer or not to participate in this portion of the study. However, we have previously shown that the genetic cohort was similar to the non-genetic cohort except for HbAlc, and we included adjustment for HbAlc in our analysis. We also did not stratify by self-identified race due to the modest cohort size, but feel that adjustment using principle components should have adequately accounted for this potential limitation.

In conclusion, using this modest sized cohort of diabetic patients newly initiated on insulin, we have identified two genomic regions as candidate loci for albuminuria and CVD disease progression. Additional investigation is warranted.

\section{References}

1. Pappachan JM, Varughese GI, Sriraman R, Arunagirinathan G (2013) Diabetic cardiomyopathy: Pathophysiology, diagnostic evaluation and management. World J Diabetes 4: 177-189.

2. Grundy SM, Benjamin IJ, Burke GL, Chait A, Eckel RH, et al. (1999) Diabetes and cardiovascular disease: a statement for healthcare professionals from the American Heart Association. Circulation 100: 1134-1146.

3. Viberti G (2003) The need for tighter control of cardiovascular risk factors in diabetic patients. J Hypertens Suppl 21: S3-6.

4. Damman K, Navis G, Voors AA, Asselbergs FW, Smilde TD, et al. (2007) Worsening renal function and prognosis in heart failure: systematic review and meta-analysis. J Card Fail 13: 599-608.

5. Donfrancesco C, Palleschi S, Palmieri L, Rossi B, Lo Noce C, et al. (2013) Estimated glomerular filtration rate, all-cause mortality and cardiovascular diseases incidence in a low risk population: the MATISS study. PLoS One 8: e78475.

6. Gerstein HC, Mann JF, Yi Q, Zinman B, Dinneen SF, et al. (2001) Albuminuria and risk of cardiovascular events, death, and heart failure in diabetic and nondiabetic individuals. JAMA 286: 421-426.

7. Jackson CE, Solomon SD, Gerstein HC, Zetterstrand S, Olofsson B, et al. (2009) Albuminuria in chronic heart failure: prevalence and prognostic importance. Lancet 374: 543-550.

8. Newman DJ, Mattock MB, Dawnay AB, Kerry S, McGuire A, et al. (2005) Systematic review on urine albumin testing for early detection of diabetic complications. Health Technol Assess 9: iii-vi, xiii-163.

9. Deshmukh HA, Palmer CN, Morris AD, Colhoun HM (2013) Investigation of known estimated glomerular filtration rate loci in patients with Type 2 diabetes. Diabet Med 30: 1230-1235.
10. Köttgen A, Pattaro C, Böger CA, Fuchsberger C, Olden M, et al. (2010) New loci associated with kidney function and chronic kidney disease. Nat Genet 42 : 376-384.

11. Liu CT, Garnaas MK, Tin A, Kottgen A, Franceschini N, et al. (2011) Genetic association for renal traits among participants of African ancestry reveals new loci for renal function. PLoS Genet 7: e1002264.

12. Ahluwalia TS, Lindholm E, Groop L, Melander O (2011) Uromodulin gene variant is associated with type 2 diabetic nephropathy. J Hypertens 29: 17311734 .

13. Ellis JW, Chen MH, Foster MC, Liu CT, Larson MG, et al. (2012) Validated SNPs for eGFR and their associations with albuminuria. Hum Mol Genet 21 3293-3298.

14. Gerstein HC, Mann JF, Yi Q, Zinman B, Dinneen SF, et al. (2001) Albuminuria and risk of cardiovascular events, death, and heart failure in diabetic and nondiabetic individuals. JAMA 286: 421-426.

15. Nathan DM, Buse JB, Davidson MB, Ferrannini E, Holman RR, et al. (2009) Medical Management of Hyperglycemia in Type 2 Diabetes: A Consensus Algorithm for the Initiation and Adjustment of Therapy: A consensus statement of the American Diabetes Association and the European Association for the Study of Diabetes. Diabetes Care 32: 193-203.

16. Ellis JW, Chen MH, Foster MC, Liu CT, Larson MG, et al. (2012) Validated SNPs for eGFR and their associations with albuminuria. Hum Mol Genet 21 3293-3298.

17. Maclsaac RJ, Panagiotopoulos S, McNeil KJ, Smith TJ, Tsalamandris C, et al (2006) Is nonalbuminuric renal insufficiency in type 2 diabetes related to an increase in intrarenal vascular disease? Diabetes Care 29: 1560-1566.

18. Amiri L, Cassidy-Bushrow AE, Dakki H, Li J, Wells K, et al. (2014) Patient characteristics and participation in a genetic study: a type 2 diabetes cohort. J Investig Med 62: 26-32.

19. Kang HM, Sul JH, Service SK, Zaitlen NA, Kong SY, et al. (2010) Variance component model to account for sample structure in genome-wide association studies. Nat Genet 42: 348-354.

20. STAMPEED: Cardiovascular Health Study GWAS to identify genetic variants associated with aging and CVD risk factors and events.

21. Cardiovascular Health Study Cohort: an NHLBI-funded observational study of risk factors for cardiovascular disease in adults 65 years or older.

22. Huang J, Ellinghaus D, Franke A, Howie B, Li Y (2012) 1000 Genomes-based imputation identifies novel and refined associations for the Wellcome Trust Case Control Consortium phase 1 Data. Eur J Hum Genet 20: 801-805.

23. Mohlke KL, Scott LJ (2012) What will diabetes genomes tell us? Curr Diab Rep 12: $643-650$ 